\title{
Attenuated innate immune defenses in very premature neonates during the neonatal period
}

\author{
Elizabeth A. Marchant ${ }^{1,2}$, Bernard Kan ${ }^{1,2}$, Ashish A. Sharma ${ }^{1,2}$, Alice van Zanten ${ }^{3,4}$, Tobias R. Kollmann ${ }^{1-4}$, Rollin Brant ${ }^{1,5}$ and \\ Pascal M. Lavoie La $^{1-4}$
}

BACKGROUND: Antimicrobial responses have been shown to be profoundly attenuated in very preterm neonates when examined on cord blood. However, we lack data on these responses at the time these neonates are most vulnerable to infections.

METHODS: Multiple cytokine responses to two prototypic Toll-like receptor (TLR) agonists: lipopolysaccharide (LPS) (TLR4) and R848 (TLR7/8) were prospectively measured in preterm neonates born $\leq 30$ wk of gestation $(n=50)$ during the first $28 \mathrm{~d}$ of age using whole blood and single-cell multiparameter flow cytometry assays. Results were compared to term neonates $(n=30)$ and adult controls $(n=25)$.

RESULTS: In preterm neonates, LPS and R848 responses remained attenuated in both cord blood and in the first $28 \mathrm{~d}$ of age. These responses showed significant maturation over time after adjusting for gestational age and were confirmed in monocytes and dendritic cells on a per-cell basis. We detected no major contribution of chorioamnionitis, maternal antenatal corticosteroids or magnesium sulfate treatment, labor, or mode of delivery to the maturation of cytokine responses.

CONCLUSION: Innate immune antimicrobial defenses are profoundly attenuated developmentally in very preterm neonates during the neonatal period, suggesting that exogenous factors drive the sustained systemic inflammation that has been linked to increased morbidities in these infants.

D espite improvements in neonatal health care, mortality from infections has continued to increase in very preterm neonates over the last two decades, owing to the improved survival of smaller and more vulnerable infants (1). However, the underlying immunological basis of their high morbidities and high vulnerability to sepsis remains poorly understood (2). To defend themselves against infection, neonates rely on first-line, innate immune defense mechanisms. These mechanisms include the detection of specific microbial molecular structures called pathogenassociated molecular patterns through specialized receptors termed pattern recognition receptors (PRR), but also other defense mechanisms such as phagocytosis and antigen presentation to the adaptive immune system.

It is widely stated that the high risk of neonatal sepsis observed in these infants is due to immature immune defense mechanisms. However, data are lacking on the development of immune functions beyond measures performed on umbilical cord blood. Recent studies have reported reduced antigen-presenting receptor expression during the first week of age in infants born below $33 \mathrm{wk}$ of gestation $(3,4)$. In contrast, phagocytic functions in these infants are more comparable to term infants (5). Toll-like receptors (TLRs) play a predominant role as a major family of PRR that act as essential "detectors" in the recognition of microbial pathogens by the innate immune system. We and others have shown that TLR-induced cytokine immune reactivity are profoundly attenuated in cord blood of very preterm neonates and develops asynchronously over the last trimester of gestation (610). These infants also display sustainably high levels of systemic inflammation, indicating a potential immune dysregulation that has also been associated with worsened clinical outcomes (11). On the other hand, cord blood responses may not adequately reflect important developmental adaptation occurring during the first few weeks after birth. Also, it is unclear to what extent specific perinatal events may induce an earlier maturation of PRR responsiveness during the neonatal period in these infants, resulting in an hyper-reactive state (12).

In this study, we determined antimicrobial TLR-induced cytokine responses in very preterm neonates over the first $28 \mathrm{~d}$ of age. We showed that these responses remain markedly attenuated during the neonatal period both as confirmed using whole blood measures and on a per-cell basis in main circulating innate immune cells. These data provide indispensable support to the premise that reduced innate immunity may contribute to the increased risk of neonatal sepsis in this age group.

\section{RESULTS}

\section{Attenuated Postnatal Immune Responses in Very Preterm} Neonates

Clinical characteristics of preterm subjects are provided in Table 1. To determine the extent to which very preterm 
Table 1. Clinical characteristics of very preterm neonates

\begin{tabular}{lc} 
Clinical characteristics & $N=50$ \\
\hline Gestational age (weeks; mean \pm SD) & $27.2 \pm 2.1$ \\
Birth weight (g; mean \pm SD) & $1,051 \pm 370$ \\
Gender $(\%$ male) $(95 \% \mathrm{Cl})$ & $67(54,81)$ \\
Received antenatal corticosteroids $(\%)(95 \% \mathrm{Cl})$ & $87(72,101)$ \\
Presence of labor $(\%)(95 \% \mathrm{Cl})$ & $70(56,83)$ \\
Received antenatal MgSO4 $(\%)(95 \% \mathrm{Cl})$ & $76(62,90)$ \\
Born by caesarian section $(\%)(95 \% \mathrm{Cl})$ & $70(56,83)$ \\
Histological chorioamnionitis $(\%)(95 \% \mathrm{Cl})$ & $49(34,63)$ \\
SNAP score at birth (median, IQR) & $26,14-49$
\end{tabular}

$\mathrm{Cl}$, confidence interval; IQR, inter-quartile range; $\mathrm{MgSO}_{4^{\prime}}$, magnesium sulfate; SNAP scores were recorded within $12 \mathrm{~h}$ of birth. neonates were able to respond to pathogen-associated molecular patterns during the neonatal period, whole blood cytokine responses to two prototypic TLR agonists were compared between very preterm neonates $(n=50)$ on both cord and postnatal blood, term infants $(n=30)$ and adult controls $(n$ $=25)$. Expectedly, cord blood LPS and R848 responses were markedly reduced in very preterm neonates (Figure 1). When comparing TLR responses in very preterm infants as a function of postnatal age, significant increase was also observed over the first $28 \mathrm{~d}$ of age (Figure 1). The distribution of gestational age in very preterm infants was identical between time periods, excluding significant effects due to the degree of gestational maturation at birth (Supplementary Figure S1 online). However, to more fully account for an effect due to gestational age, postnatal TLR reactivity was analyzed in very preterm

a
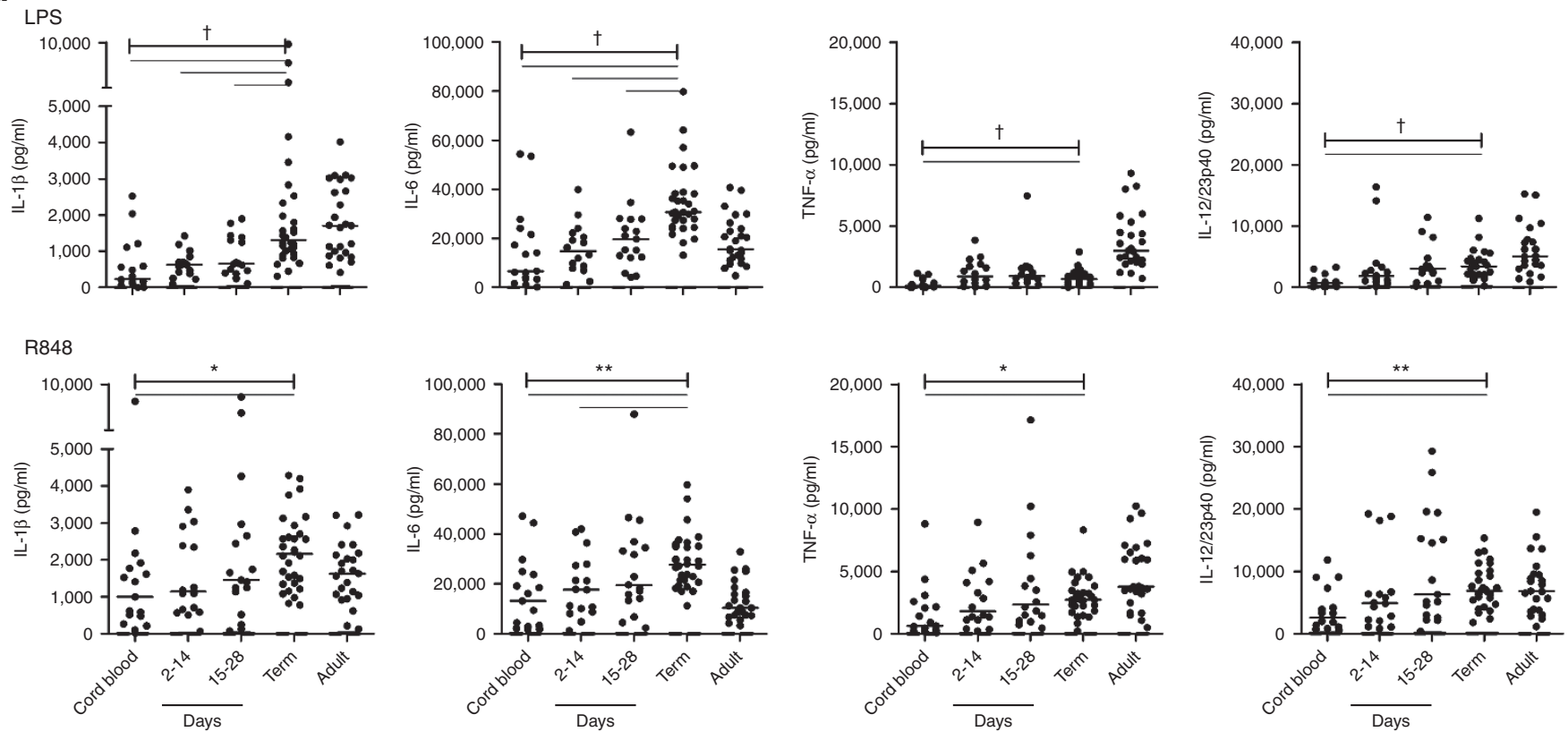

b
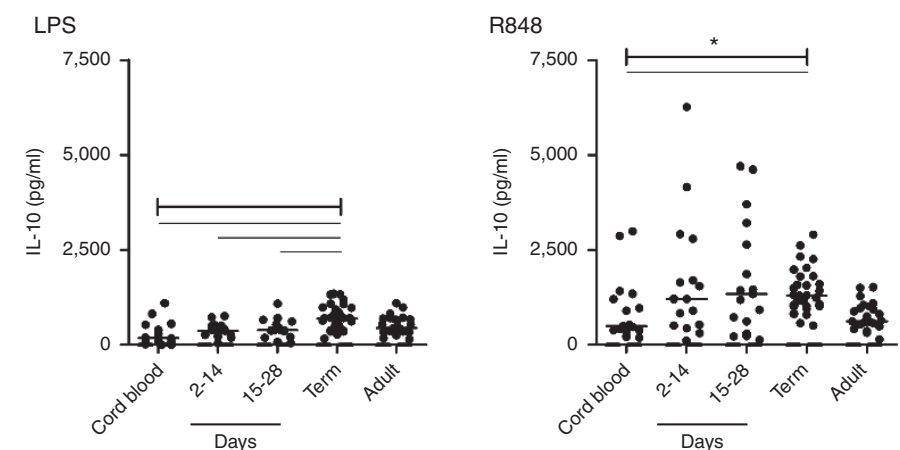

Figure 1. Toll-like receptor cytokine responses. Whole blood (a) pro- and (b) anti-inflammatory cytokine responses in very preterm neonates grouped by sampling period on cord blood, 2-14 and 15-28 d of age, and in term neonates and adults, following stimulation with lipopolysaccharide or R848 (bars represent median). Unstimulated cytokine levels (median) were undetectable ( $\leq 0 \mathrm{pg} / \mathrm{ml}$ ) in all cases (not shown). Significant differences in cytokine responses across all three groups of very preterm neonates and term neonates, as determined using an ANOVA, are shown as a capped-end line $\left({ }^{*} P<0.05 ;{ }^{*} P<0.01 ;{ }^{+} P<0.001\right)$. Statistical comparisons within each subgroup, as determined using a Dunn's post-test taking into account multiple comparisons, is shown as straight lines $(P<0.05)$. 
neonates according to postnatal days used as a continuous variable, in regression models. Estimates of effects are presented in Table 2 and quantify the increase in TLR responsiveness for each increase in postnatal days independent of gestational age, within the group of very preterm neonates. These results confirmed a large effect of postnatal age on most LPS and R848 cytokine responses. Indeed, significant residual influences of postnatal age were detected for IL-6 responses (using R848), TNF- $\alpha$ (for both LPS and R848) and IL-12/23p40 (for both LPS and R848) $(P<0.05)$.

TLR Reactivity at the Single-Cell Level in Very Preterm Neonates To ascertain that the attenuated TLR reactivity was not due to a lack of circulating immune cells in whole blood, cell compositions were enumerated using flow cytometry. Monocyte proportions were substantially higher in very preterm neonates during the neonatal period, compared to term neonates, adults, or even compared to preterm cord blood samples, whereas proportions of granulocytes, lymphocytes and dendritic cells were comparable (Supplementary Figure S2 online).

To further exclude a lack of cytokine-producing cells, TLRinduced production of IL-6, TNF- $\alpha$, and p40, a molecular subunit shared between the IL-12 and IL-23 cytokines, were also analyzed at the single cell level by intracellular cytokine flow cytometry detection in two main cytokine-producing innate immune blood cell types: CD14-expressing monocytes and CD11c-expressing dendritic cells. A representative flow cytometry diagram of intracellular cytokine responses from a representative very preterm infant is shown in Supplementary Figure S3 online). As shown in Figure 2, production of IL-6 and TNF- $\alpha$ was substantially reduced in a subgroup of very preterm infants both in monocytes, compared to term neonates or adults. Dendritic cells are by and large the main source of IL-12/23p40 in human blood. These two cytokines are important in the differentiation of $\mathrm{T}$ helper lymphocyte immune responses (7). Similar to the reduced IL-6 and TNF$\alpha$ responses observed in preterm monocytes, production of

Table 2. Changes in TLR responses by postnatal age, adjusted for gestational age

\begin{tabular}{llcl}
\hline TLR ligand & Cytokine & $\begin{array}{c}\text { Estimates of effects } \\
(95 \% \mathrm{Cl})\end{array}$ & $P$ value \\
\hline Lipopolysaccharide & IL-1 $\beta$ & $742(-140,1,631)$ & 0.107 \\
& IL-6 & $21301(-1,036,43,637)$ & 0.068 \\
& TNF- $\alpha$ & $2450(560,4,340)$ & $0.014^{*}$ \\
& IL-12/23p40 & $6076(770,11,382)$ & $0.029^{*}$ \\
& IL-10 & $364(-21,756)$ & 0.069 \\
R848 & IL-1 $\beta$ & $2779(-210,5,775)$ & 0.075 \\
& IL-6 & $36253(10,675,61,838)$ & $0.008^{* *}$ \\
& TNF- $\alpha$ & $76321(490,12,663)$ & $0.005^{* *}$ \\
& IL-12/23p40 & $17703(6,790,28,616)$ & $0.003^{* *}$ \\
& IL-10 & $1939(-308,4,186)$ & 0.098 \\
\hline$* P<0.05 ; * * 0<0.01$. & & & \\
TLR, Toll-like receptor. & & &
\end{tabular}

IL-12/23p40 was also markedly reduced in dendritic cells from both term and preterm neonates (Figure 2). Collectively, these data confirm that innate immune cells are present in preterm neonates but further indicate that these cells are hyporeactive to TLR stimulation in vitro.

\section{Contribution of Perinatal Factors to the Maturation of Postnatal Innate Immune Responses}

To assess whether responses were independently influenced by common perinatal factors linked to prematurity (including chorioamnionitis; antenatal corticosteroids; presence of labor; mode of delivery of the infant; and use of magnesium sulfate), the contribution of these factors was examined in the group of very preterm neonates using partial correlations, adjusting for gestational age. As shown in Figure 3, none of the factors had significant effects on the ontogeny of innate cytokine production, indicating a developmental maturation rather than attenuation due to exogenous perinatal factors.

\section{DISCUSSION}

To our knowledge, this is the first study that measured antimicrobial reactivity in very preterm neonates during the neonatal period. We confirm profoundly attenuated post-natal responses, consistent with responses measured on cord blood $(7,8)$. The use of single-cell flow cytometry allows confirmation that the functional attenuation in TLR responsiveness is not due to a lack of innate immune cell composition. The measure of responses on whole blood provides a more global determination of anti-microbial responses. Indeed, previous studies have demonstrated that neonatal plasma suppresses TLR-mediated cytokine production, indicating that both cellular and soluble humeral factors are important in the functional determination of immune responsiveness $(13,14)$.

Our data are consistent with previous studies that have measured a low surface expression of TLRs on blood mononuclear cells of preterm neonates, with TLR2 and TLR4 expression increasing during the first 2 mo of age (15). However, because TLR-dependent cytokine responses generally correlate poorly with their corresponding receptor gene or protein expression, it is important to validate the impact of a reduced expression of PRR at the functional level by examining relevant cytokine responses (6). Others reported that endotoxin (TLR4) responses in monocytes from preterm neonates born below $32 \mathrm{wk}$ of gestation are already mature (i.e., comparable to responses measured at the full term of gestation) by the second week of age, although it remained unclear whether this is also true of infants born at the lower end of gestation (15).

Our data help address an important paradox in neonatal research. High circulating levels of inflammatory cytokines have been observed in very preterm infants exposed to intensive care (11) and have been repeatedly linked to worse outcomes such as bronchopulmonary dysplasia (12), white matter injury, and neurodevelopmental impairments (16). These results have led to speculations that the innate immune system of these infants is hyper- rather than hypo-responsive during the neonatal period (17). However, this hypothesis was 
a

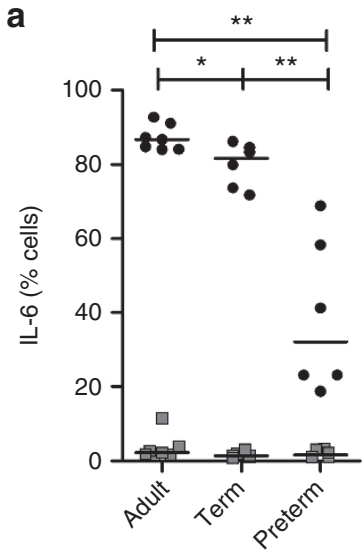

b

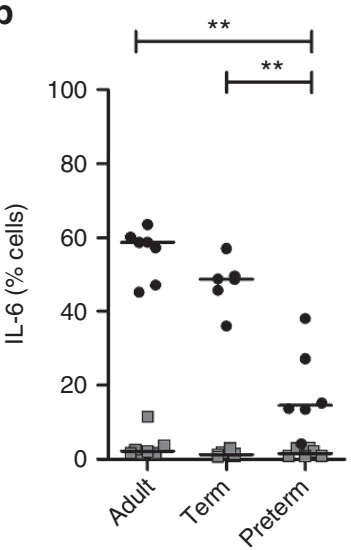

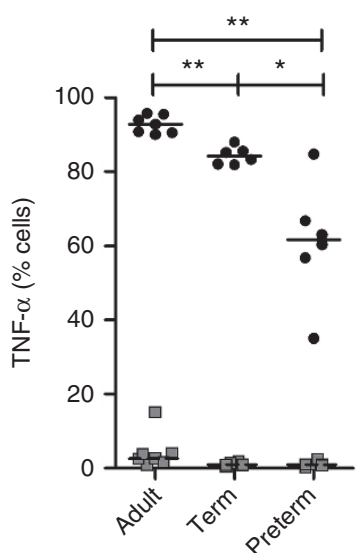
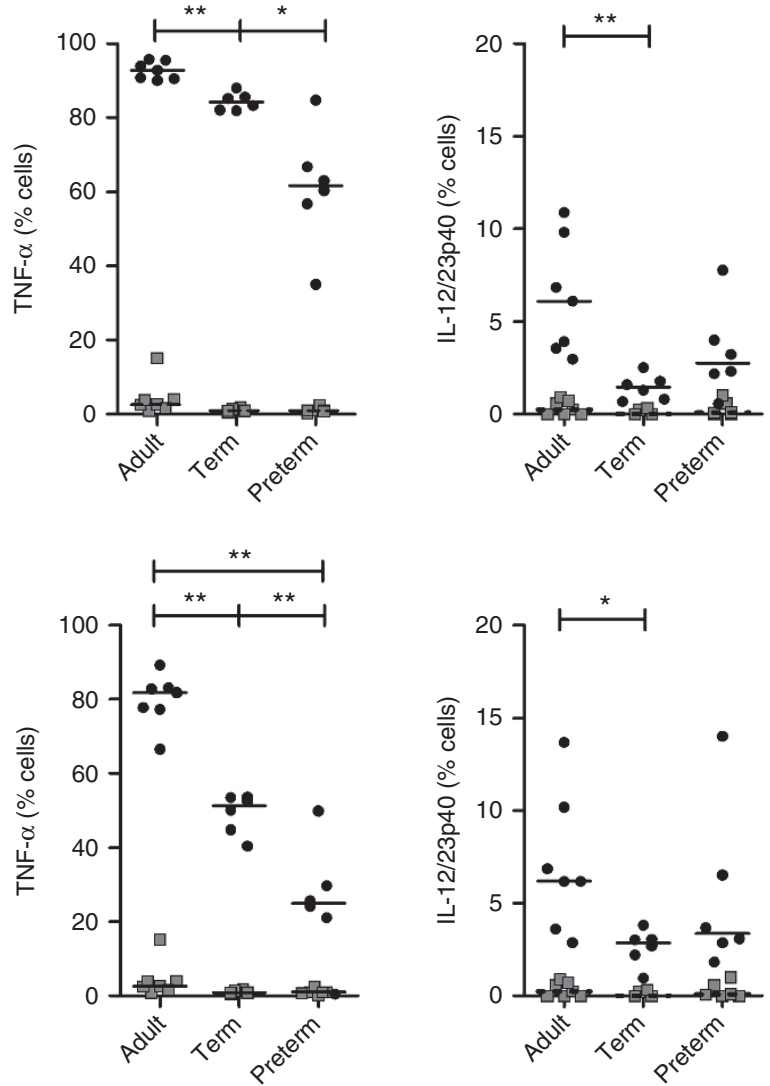

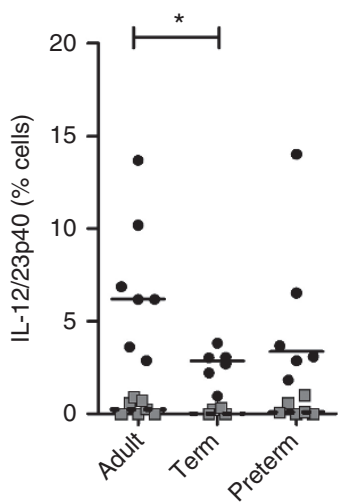

Figure 2. Single-cell cytokine production in innate immune cells. Single-cell (a) lipopolysaccharide - and (b) R848-induced cytokine responses (black circles) in monocytes (for IL-6 and TNF- $\alpha$ ) and in dendritic cells (for IL-12/23p40) in very preterm neonates (mean gestational age and birth weight: $27.2 \mathrm{wk}$ and 1,063 g, respectively; median postnatal age: $13 \mathrm{~d}$, range 7-23), term neonates and adults, compared to unstimulated responses (gray squares). ${ }^{*} P<0.05 ;{ }^{* *} P<0.01$

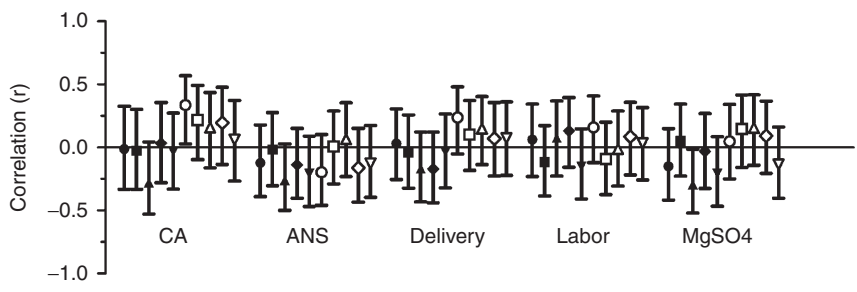

Figure 3. Effect of perinatal factors on the postnatal maturation of Tolllike receptor responses. Data show correlations (y-axis) adjusted for gestational age, with $95 \%$ confidence intervals (bars). Lipopolysaccharide (black symbols) and R848 (white symbols) responses by cytokine; IL-1 $\beta$ (circle), IL-6 (square), TNF- $\alpha$ (upward triangle), IL-12/23p40 (diamond), IL-10 (downward triangle). ANS, antenatal corticosteroids; CA, chorioamnionitis.

based mainly on random measures of inflammatory mediators in serum, and had, to the best of our knowledge, never been confirmed by functional data measuring the "reactivity" of the postnatal innate immune system. In fact, studies using cord blood have consistently shown that the innate immune defenses of the very preterm infants are hyporeactive to PRR stimulation, compared to term-born infants or adults. Our data extend these observations, namely that very preterm neonates continue to display decreased TLR-agonist induced production of both pro-inflammatory (IL-1 $\beta$, IL-6, TNF- $\alpha$,
IL-12/23p40) and regulatory (IL-10) cytokines over the entire first month of life. Also, previous cord blood studies have suggested that immune cells from very preterm neonates may in fact be biased toward an anti-inflammatory state characterized by higher IL-10 production compared to other proinflammatory cytokine responses (7). However, this was not observed in our current study. This then raises the question: why are basal levels of inflammation elevated in very preterm neonates during the neonatal period? A possible explanation is that the high basal inflammation reflects an ongoing, continuous exposure to stimulation in the context of repeated oxidative stress due to mechanical ventilation, supplemental oxygen or sepsis. Additionally, the high circulating levels of systemic inflammation in these infants may reflect impaired mechanisms of active resolution of the inflammation rather than a sustained activation. The possibility that worse outcomes may result from a premature immune maturation of antimicrobial responses does, however, raise potential concerns of using experimental therapies aiming to "boost" immune reactivity at an earlier stage, in order to prevent neonatal infections in this age group.

TLRs play a critical role in protecting against microbes in early life, as indicated by an increased vulnerability to specific bacterial and viral infections in humans with loss-of-function 
mutations along these molecular pathways (18). The risk of infections in these patients is greatest during the neonatal/early childhood period, underlining the importance of these receptors for immune protection during infancy $(18,19)$. As shown from previous epidemiological studies, the incidence of sepsis sharply increases in these infants shortly after birth, peaking during the first week of postnatal age, then slowly declining thereafter (20,21). Although additional reasons may underlie the increased vulnerability of these infants to sepsis, including a high need for invasive life-saving interventions early on, and the prompt microbial colonization of their skin and mucosal membranes after birth, our data mirror this period of vulnerability. This window of vulnerability temporally delineates a high risk period during which exposure to skin- or mucosalbreaking invasive interventions should be insistently minimized, in order to limit the risk of sepsis. Overall, these data provide essential support to the premise that the increased risk of infection in very preterm neonates is due to a developmental immaturity of innate immune anti-microbial responses.

Some limitations to our study should be acknowledged. Due to a limited number of preterm infants enrolled, we were unable to determine the relationship between attenuated antimicrobial responses and an increased risk of sepsis, or other clinical outcomes. Also, we were unable to detect an effect of perinatal factors, (i.e., chorioamnionitis, antenatal corticosteroids, exposure to magnesium sulfate, presence of labor, etc.) on the maturation of innate immune responses in the group of very preterm infants. Although our analysis was not specifically powered to detect subtle effects, again due to a limited sample size, we can exclude large effects related to an exposure to antenatal corticosteroids or chorioamnionitis that could be implied from animal models $(22,23)$. Our study also highlights the importance of corroborating observation in populations of human infants, even if ethically challenging, in order to validate animal observations. Moreover, our study certainly informs as to the considerably larger sample size that would be required in order to detect more subtle effects from perinatal factors on the maturation of innate immune functions.

In conclusion, we show that the ability of very preterm neonates to respond to pathogen-associated molecular patterns is profoundly attenuated over the first month after birth in very preterm neonates, yet with considerable heterogeneity in the degree and timing of post-natal maturation. Our data are directly relevant to our understanding of how and when immaturity of the developing innate immune system contributes to an increased risk of sepsis in these infants. Future studies are required to understand how variability in immune responses among individuals affects clinical outcomes.

\section{METHODS}

\section{Study Population}

Neonates were prospectively enrolled at the British Columbia Children's \& Women's Health Centre (Vancouver, Canada) between June 2012 and March 2014, after parental informed consent. Preterm neonates born below $30 \mathrm{wk}$ of gestation admitted to the Neonatal Intensive Care Unit were sampled once, according to a gestational age-matched schedule where blood was taken during either periods of 2 to 14 , or 15 to $28 \mathrm{~d}$ of age in order to distribute subjects evenly across postnatal days. All peripheral blood samples were coordinated with other early morning routine blood taking. Also, samples were scheduled to be collected at least $48 \mathrm{~h}$ away from a blood product transfusion, or from postnatal corticosteroid administration. Infants who were actively diagnosed with suspected or proven sepsis or under antibiotic treatment were excluded. For external validation, the amount of exposure to supplemental oxygen, mechanical ventilation, parenteral nutrition, and use of intravenous sedation (either morphine or midazolam) at time of blood sampling in preterm neonates is also provided in Supplementary Table S1 online). To further confirm generalizability of our findings, white blood cell composition was determined using a diagnostic automated cell count for neutrophils, monocytes and lymphocytes in the subgroup of very preterm neonates throughout the study period, as part of routine clinical care and were within expected normal ranges (see Supplementary Figure S4 online). Cord blood samples were also obtained from healthy control neonates delivered at term by elective Caesarean section and from peripheral blood of healthy adults using identical protocols and reagent batches. All blood samples were collected in sodium heparin $\mathrm{BD}$ Vacutainer tubes (Becton, Dickinson, Mississauga, Canada) and processed within $1 \mathrm{~h}$ of collection. To avoid an experimental historical bias during recruitment, a proportional number of preterm, term, and adult subjects were tested over the course of the study. Chorioamnionitis was defined histologically as previously described (11). Antenatal corticosteroid and magnesium sulfate exposure were defined as occurring within 72 and $24 \mathrm{~h}$ prior to delivery, respectively. SNAP scores were determined as described (24). The study was approved by the University of British Columbia Children's \& Women's Research Ethics Board (\#H07-02681).

\section{Immune Stimulation and Cytokine Measures}

To limit experimental variability in our assays, TLR agonists were prediluted in batches at the beginning of the study in quantities sufficient for 6-mo periods in accordance with prestudy stability tests done of all main reagents used in the study (data not shown). To ensure stability of the reagents, each ligand batch was also tested against a group of adult subjects at regular intervals (not shown). Whole blood was diluted 1:2 with RPMI 1640 medium with L-glutamine (Gibco, Grand Island, NY) and stimulated with saturating doses of the TLR4 agonist lipopolysaccharide (LPS, $100 \mathrm{ng} / \mathrm{ml}$; InvivoGen, San Diego, CA), the TLR7/8 agonist R848 ( $1 \mu \mathrm{g} / \mathrm{ml}$; InvivoGen), or unstimulated, for $24 \mathrm{~h}$ at $37^{\circ} \mathrm{C}$ in $5 \% \mathrm{CO}_{2}$ atmosphere in prediluted aliquots round-bottom 96-well microplates. The concentration of TLR agonists used in this study corresponded to peak stimulating concentrations, as determined in our previous studies $(7,25)$. Following stimulation, cell supernatants were stored at $-80^{\circ} \mathrm{C}$ for batches analyses. Cytokine measurements were performed in duplicates using commercially available enzyme-linked immunosorbent assays (ELISA) for the following cytokines: IL-1 $\beta$, IL-6, IL-10, and TNF- $\alpha$ (eBioscience, San Diego, CA) and IL-12p40 (BioLegend, San Diego, CA), as described (7). Mean intra-assay coefficients of variability for all cytokine-stimulated levels consistently were below $15 \%$. In each age group, half of the subjects were also randomly sampled in parallel for use in whole blood immune cell composition and intracellular cytokine production assays, using flow cytometry as described (7). For flow cytometry analyses, the following antibodies were used: Alexa Fluor 700-conjugated anti-CD11c, PE-Cy7-conjugated anti-CD14, eVolve605-conjugated anti-CD19 and PerCP-Cy5.5conjugated anti-HLA-DR (eBioscience), and PE-conjugated anti-CD3 (BD Biosciences, Mississauga, Canada) for identification of monocytes (HLA-DR, CD14), myeloid dendritic cells (HLA-DR, CD11c), B cells (CD19), T cells (CD3), and polymorphonuclear cells (by forward and side scatter), as described (7). For intracellular cytokine detection, cells were incubated in presence of brefeldin A $(10 \mu \mathrm{g} / \mu \mathrm{l}$, Sigma Aldrich, St. Louis, MO) and the following additional antibodies were used (after permeabilization): Pacific Blue-conjugated anti-CD3 (instead of PE-conjugated, BioLegend), APC-conjugated or FITC-conjugated antiIL-6, PE-eFluor610-conjugated TNF- $\alpha$ and PE-conjugated-IL-12/23p40 (eBioscience), as described (7). No significant cytokine production was detected in unstimulated polymorphonuclear cells (not shown). In all flow cytometry analyses, cells were also stained using FITC-conjugated 
anti-glycophorin A to allow gating out of any residual red blood cells in the sample and gates were adjusted using fluorescence-minus one controls (for cell surface markers) and unstimulated control samples (for cytokine detection). Data were analyzed using the FlowJo software (FlowJo vX 10.0.7r2; FlowJo LLC, Ashland, OR).

\section{Statistical Analyses}

Differences between stimulated and baseline cytokine responses were compared between age groups using nonparametric testing (Mann-Whitney $U$ ) and were all highly significant $(P<0.0001$; data not shown). In initial analyses, responses within the whole group of preterm neonates were assessed using linear regressions and were shown to be independent of whether samples were collected at the time of birth from cord blood (labeled as day 1), or from postnatal peripheral blood. This finding was then confirmed using analysis of covariance incorporating postnatal age as a continuous variable, where cytokine responses were then compared between cord and peripheral blood (data not shown); therefore all cord and peripheral blood samples were combined in subsequent analyses. To determine the impact of postnatal age, differences between groups were compared using a one-way ANOVA, as a function of postnatal age (categorized as preterm cord blood, preterm day 2-14 and preterm day 15-28, term) and using a post-test Dunn's correction for multiple testing $(P<0.05)$. In subsequent analyses, the relative contributions of gestational age and the independent effect of postnatal age were confirmed within the subgroup of very preterm neonates, using multiple linear regressions where both parameters (gestational and postnatal age) were included as continuous variables. The residual contributions of perinatal factors (chorioamnionitis: yes/no, antenatal corticosteroids: yes/no, presence of labor: yes/no, mode of delivery of the infant: vaginal/C-section and use of magnesium sulfate: yes/no) were individually assessed using partial correlations, with adjustment for gestational age (as a continuous variable). Associated $95 \%$ confidence intervals were obtained using the bootstrap.

\section{SUPPLEMENTARY MATERIAL}

Supplementary material is linked to the online version of the paper at http://www.nature.com/pr

\section{ACKNOWLEDGMENTS}

We thank Jennifer Claydon for coordination of the study, Kristi Finlay and the BC Women's Hospital delivery room staff for help with recruitment of subjects, and Rosemary Delnavine for editorial revisions.

\section{STATEMENT OF FINANCIAL SUPPORT}

This research was supported by grants from the Canadian Institutes of Health Research (MOP-110938) and a Gene Discovery and Translational Research Grant No. 6-FY12-315 from the March of Dimes Foundation. P.M.L. received salary support from a Clinician-Scientist Award by the Child \& Family Research Institute (CFRI) and a Career Investigator Award from the Michael Smith Foundation for Health Research. E.A.M. and A.A.S. were supported by studentships from CFRI.

Disclosure: The authors have no financial disclosures and no conflicts of interest to declare.

\section{REFERENCES}

1. Berrington JE, Hearn RI, Bythell M, Wright C, Embleton ND. Deaths in preterm infants: changing pathology over 2 decades. J Pediatr 2012;160: 49-53.e1.

2. Sharma AA, Jen R, Butler A, Lavoie PM. The developing human preterm neonatal immune system: a case for more research in this area. Clin Immunol 2012;145:61-8.

3. Azizia M, Lloyd J, Allen M, Klein N, Peebles D. Immune status in very preterm neonates. Pediatrics 2012;129:e967-74.

4. Palojärvi A, Andersson S, Turpeinen U, Janér C, Petäjä J. Antenatal betamethasone associates with transient immunodepression in very low birth weight infants. Neonatology 2013;104:275-82.
5. Prosser A, Hibbert J, Strunk T, et al. Phagocytosis of neonatal pathogens by peripheral blood neutrophils and monocytes from newborn preterm and term infants. Pediatr Res 2013;74:503-10.

6. Strunk T, Prosser A, Levy O, et al. Responsiveness of human monocytes to the commensal bacterium Staphylococcus epidermidis develops late in gestation. Pediatr Res 2012;72:10-8.

7. Lavoie PM, Huang Q, Jolette E, et al. Profound lack of interleukin (IL)-12/ IL-23p40 in neonates born early in gestation is associated with an increased risk of sepsis. J Infect Dis 2010;202:1754-63.

8. Sharma AA, Jen R, Brant R, et al. Hierarchical maturation of innate immune defences in very preterm neonates. Neonatology 2014;106:1-9.

9. Förster-Waldl E, Sadeghi K, Tamandl D, et al. Monocyte toll-like receptor 4 expression and LPS-induced cytokine production increase during gestational aging. Pediatr Res 2005;58:121-4.

10. Sadeghi K, Berger A, Langgartner M, et al. Immaturity of infection control in preterm and term newborns is associated with impaired toll-like receptor signaling. J Infect Dis 2007;195:296-302.

11. Chang BA, Huang Q, Quan J, et al. Early inflammation in the absence of overt infection in preterm neonates exposed to intensive care. Cytokine 2011;56:621-6.

12. Paananen R, Husa AK, Vuolteenaho R, Herva R, Kaukola T, Hallman M. Blood cytokines during the perinatal period in very preterm infants: relationship of inflammatory response and bronchopulmonary dysplasia. J Pediatr 2009;154:39-43.e3.

13. Belderbos ME, Levy O, Stalpers F, Kimpen JL, Meyaard L, Bont L. Neonatal plasma polarizes TLR4-mediated cytokine responses towards low IL-12p70 and high IL-10 production via distinct factors. PLoS One 2012;7:e33419.

14. Levy O, Zarember KA, Roy RM, Cywes C, Godowski PJ, Wessels MR. Selective impairment of TLR-mediated innate immunity in human newborns: neonatal blood plasma reduces monocyte TNF-alpha induction by bacterial lipopeptides, lipopolysaccharide, and imiquimod, but preserves the response to R-848. J Immunol 2004;173:4627-34.

15. Shen CM, Lin SC, Niu DM, Kou YR. Development of monocyte Toll-like receptor 2 and Toll-like receptor 4 in preterm newborns during the first few months of life. Pediatr Res 2013;73:685-91.

16. Strunk T, Inder T, Wang X, Burgner D, Mallard C, Levy O. Infectioninduced inflammation and cerebral injury in preterm infants. Lancet Infect Dis 2014;14:751-62.

17. Wright CJ, Kirpalani H. Targeting inflammation to prevent bronchopulmonary dysplasia: can new insights be translated into therapies? Pediatrics 2011;128:111-26.

18. Picard C, von Bernuth $\mathrm{H}$, Ghandil P, et al. Clinical features and outcome of patients with IRAK-4 and MyD88 deficiency. Medicine (Baltimore) 2010;89:403-25.

19. Currie AJ, Davidson DJ, Reid GS, et al. Primary immunodeficiency to pneumococcal infection due to a defect in Toll-like receptor signaling. J Pediatr 2004;144:512-8.

20. Lavoie PM. Earlier initiation of enteral nutrition is associated with lower risk of late-onset bacteremia only in most mature very low birth weight infants. J Perinatol 2009;29:448-54.

21. Isaacs D; Australasian Study Group For Neonatal Infections. A ten year, multicentre study of coagulase negative staphylococcal infections in Australasian neonatal units. Arch Dis Child Fetal Neonatal Ed 2003;88:F89-93.

22. Kramer BW, Ikegami M, Moss TJ, Nitsos I, Newnham JP, Jobe AH. Endotoxin-induced chorioamnionitis modulates innate immunity of monocytes in preterm sheep. Am J Respir Crit Care Med 2005;171:73-7.

23. Newnham JP, Kallapur SG, Kramer BW, et al. Betamethasone effects on chorioamnionitis induced by intra-amniotic endotoxin in sheep. Am J Obstet Gynecol 2003;189:1458-66.

24. Richardson DK, Corcoran JD, Escobar GJ, Lee SK. SNAP-II and SNAPPEII: Simplified newborn illness severity and mortality risk scores. J Pediatr 2001;138:92-100.

25. Jansen K, Blimkie D, Furlong J, et al. Polychromatic flow cytometric highthroughput assay to analyze the innate immune response to Toll-like receptor stimulation. J Immunol Methods 2008;336:183-92. 